\title{
ARTÍCULOS
}

Sometido 20.01.2016. Aprobado 28.06.2016

Evaluado por el sistema double blind review. Editor Científico: Diogo Helal

DOI: http://dx.doi.org/10.1590/So034-759020170202

\section{EL EFECTO DE LA DIVERSIDAD DE GÉNERO Y EL NIVEL EDUCATIVO EN LA INNOVACIÓN}

\author{
O efeito da diversidade de gênero e o nível educacional na inovação \\ The effect of gender diversity and education level on innovation
}

\section{ANA M. ROMERO-MARTÍNEZ \\ amromero@ucm.es \\ Profesora de la Universidad \\ Complutense de Madrid, Facultad de \\ Ciencias Económicas y Empresariales \\ - Madrid, España}

\section{ÁNGELES MONTORO-SÁNCHEZ \\ mangeles@ccee.ucm.es \\ Profesora de la Universidad \\ Complutense de Madrid, Facultad de \\ Ciencias Económicas y Empresariales \\ - Madrid, España}

\section{YOUSELINE GARAVITO-HERNÁNDEZ}

yougaravito@gmail.com

Profesora de la Universitaria

de Investigación y Desarrollo -

Bucaramanga, Colombia

\section{RESUMEN}

El objetivo de este trabajo es analizar el impacto de la diversidad de género y el nivel educativo de los empleados de I+D en la innovación. Para ello, empleamos como fuente de información la encuesta de innovación tecnológica en España, que pertenece al plan general de estadísticas de la Unión Europea (Eurostat). Con una muestra de 7.962 empresas, en su mayoría pequeñas, los resultados revelan que la diversidad de género y el nivel educativo de los empleados de I+D influyen positivamente de forma individual en la innovación de producto, aunque no se encuentra un impacto significativo del efecto conjunto.

PALABRAS CLAVE | Innovación, diversidad de género, nivel educativo, l+D, innovación de producto.

\section{RESUMO}

O objetivo deste trabalho é analisar o impacto da diversidade de gênero e do nível educacional dos funcionários da I+D na inovação. Para tanto, empregamos como fonte de informação a pesquisa de inovação tecnológica na Espanha, que faz parte do plano geral de estatísticas da União Europeia (Eurostat). A partir de uma amostra de 7.962 empresas - em sua maioria, pequenas -, os resultados revelam que a diversidade de gênero e o nível educacional dos funcionários da $1+D$ influenciam positivamente, de forma individual, a inovação de produto, embora não se encontre uma influência significativa do efeito conjunto.

PALAVRAS-CHAVE / Inovação, diversidade de gênero, nível educativo, I+D, inovação de produto.

\section{ABSTRACT}

The aim of this paper is to analyze the impact of gender diversity and education level of R\&D employees on innovation. We use as source of information the Community Innovation Survey belonging to the general plan for statistics of the European Union (Eurostat). From a sample of 7,962 firms, most of them small firms, the results show that the diversity of gender and educational level of $R \& D$ employees individually have a positive influence on product innovation, although there is no significant effect of combined effect.

KEYWORDS / Innovation, gender diversity, educational level, R\&D, product innovation. 


\section{INTRODUCCIÓN}

La innovación es clave en la supervivencia de las empresas ya que para competir en los actuales entornos, cada vez más cambiantes, éstas han de crear nuevos productos y servicios (Cefis \& Marsili, 2006; Velu, 2015). Por ello, durante las últimas décadas uno de los tópicos más estudiados han sido los factores que influyen en la innovación, tales como el tamaño de la empresa, la industria, los recursos financieros, la cooperación en I+D, los recursos tecnológicos y el capital humano (Donate \& Guadamillas, 2011; Sarooghi, Libaers, \& Burkemper, 2015). Respecto a éste último, los trabajos más recientes analizan factores como la cultura organizativa, el liderazgo, la diversidad y el nivel educativo de los empleados; y, entre ellos, la diversidad de género y el nivel educativo de los empleados son los que reclaman mayor atención (Crawford, Gould, \& Scott, 2003; DeLong \& Fahey, 2000; DíazGarcía, González-Moreno, \& Sáez-Martínez, 2013; Østergaard, Timmermans, \& Kristinsson, 2011).

En cuanto a la diversidad de género, ésta se define como el grado de heterogeneidad o diferencias en la composición de un grupo de individuos en función del sexo (Kearney, Gebert, \& Voelpel, 2009; Simons, Pelled, \& Smith, 1999). Diversos autores señalan que la diversidad de género se asocia positivamente con la innovación (Díaz-García et al., 2013; Doorn, Jansen, Bosch, \& Volberda, 2013; Horwitz, 2005; Jehn, Northcraft, \& Neale, 1999). Sin embargo, la mayoría de estos trabajos evalúan este efecto sólo en equipos de alta dirección, salvo casos excepcionales como Østergaard et al. (2011) que lo analiza a nivel de empresa, Fernández-Sastre (2015) en los equipos de I+D y Díaz-García et al. (2013) en los empleados de I+D, lo que constata la necesidad de mayor evidencia empírica.

Al igual que la diversidad de género, el nivel educativo constituye un recurso fundamental para el desarrollo de nuevas ideas y para alcanzar el éxito en las iniciativas innovadoras que persigue la empresa (Vegt \& Janssen, 2003). Las actividades de innovación requieren creatividad y conocimientos técnicos y especializados, y son los empleados con alto nivel formativo los que los tienen (Hayton \& Kelley, 2006; Oldham \& Cummings, 1996). Una evidencia empírica previa muestra el efecto positivo y significativo de la educación en la innovación (Alexiev, Jansen, Bosch, \& Volberda, 2010; Bantel \& Jackson, 1989; Heavey \& Simsek, 2013; Wiersema \& Bantel, 1992; Wu, Lin, \& Hsu, 2007). Al igual que en la diversidad de género, los trabajos se han centrado fundamentalmente en los equipos de la alta dirección. Por ello, se necesita mayor evidencia empírica a nivel de la empresa y, especialmente, en el caso de los empleados de I+D (Alexiev et al., 2010; Corbett, Covin, O’Connor, \& Tucci, 2013; Heavey \& Simsek, 2013).
Finalmente, una relación novedosa y que no se ha estudiado hasta el momento es el efecto conjunto de la diversidad de género y el nivel educativo de los empleados de I+D en la innovación. La heterogeneidad de género con alto nivel formativo está cada vez más presente en las empresas (Love \& Roper, 2015) y por lo tanto, puede influir en su capacidad innovadora. La mayor incorporación de la mujer al mundo laboral y con el mismo nivel de estudios que los hombres hace que en muchos casos adopten estereotipos masculinos que se asocian fuertemente con rasgos y comportamientos de mayor contenido innovador (Gupta, Goktan, \& Gunay, 2014). Por todo ello, es relevante demostrar el potencial efecto positivo del alto nivel educativo de los empleados, especialmente de los de I+D que tienen un papel clave en el desarrollo de productos, en la relación entre la diversidad de género y la innovación (Bruin, Brush, \& Welter, 2007).

Por lo tanto, el objetivo de este trabajo es estudiar el efecto de la diversidad de género y el nivel educativo de los empleados de I+D, por separado y conjuntamente, en la innovación empresarial. Con ello, este trabajo realiza las siguientes contribuciones: En primer lugar, permite profundizar en la relación entre la diversidad de género, el nivel educativo y la innovación de la empresa, dentro de la literatura de innovación. Dado que la creación de valor de la empresa resultado de su posición competitiva no sólo depende del comportamiento de la alta dirección, sino del hacer de todo el capital humano de la empresa (Alexiev et al., 2010), este trabajo contribuye a la literatura previa analizando estos factores en los empleados de I+D, frente a la gran mayoría de trabajos previos que ponen el foco sólo en los equipos de alta dirección. En segundo lugar, este trabajo también supone una contribución a la literatura de recursos humanos, en la cual también son escasos los trabajos que estudian a los empleados de I+D desde la perspectiva del capital humano. No obstante, existen algunos trabajos centrados en motivación (Manolopoulos, 2006), liderazgo, clima, intercambio de conocimiento (Liu \& Liu, 2011) y especialmente cabe destacar el trabajo de Zhang y Begley (2011), que estudia clima organizativo, transferencia de conocimiento e innovación en los profesionales de I+D. En tercer lugar, el presente estudio avanza en el análisis del estudio de estos factores dando un paso más al evaluar el potencial efecto conjunto de la diversidad de género y la educación en la innovación, aspecto de gran novedad que previamente no se ha considerado en la literatura existente, y que puede arrojar más luz sobre el efecto de estos dos factores del capital humano en la innovación empresarial. Adicionalmente, respecto a la evidencia empírica que se aporta, las características de la muestra empleada constituyen también otra importante aportación. El contraste empírico de este trabajo se realiza en una muestra de empresas españolas. Esto supone una aportación en la medida 
en que la mayor parte de la evidencia existente corresponde a muestras de empresas especialmente de Estados Unidos, o de otros países como Irlanda, Holanda, Dinamarca y Taiwán (Alexiev et al., 2010; Bantel \& Jackson, 1989; Hayton, 2005; Heavey \& Simsek, 2013; Østergaard et al., 2011). Además, dada la situación de crisis generalizada de los últimos años dentro de Europa, de España en particular, evaluar cómo ambos aspectos del capital humano afectan a la innovación en la empresa, y por ende a la supervivencia de las empresas, resulta de gran interés de cara a posibles políticas gubernamentales que puedan ayudar a potenciar la diversidad de género y el nivel formativo de los empleados. Finalmente, la muestra de empresas de este trabajo reúne tanto el sector manufacturero como el sector servicios, mientras que estudios previos suelen emplear muestras de sectores manufactureros o centrarse sólo en sectores de alta tecnología (Phan, Wright, Ucbasaran, \& Tan, 2009).

Para alcanzar el objetivo de investigación señalado, el artículo se estructura de la siguiente manera: en primer lugar, se expone la revisión de la literatura y se justifican las hipótesis planteadas. A continuación se muestra la metodología, con información sobre la muestra utilizada y las medidas de las variables. Después se presentan los resultados obtenidos. Por último, se exponen la discusión y las conclusiones.

\section{MARCO TEÓRICO E HIPÓTESIS DE TRABAJO}

En los últimos años, el estudio de la diversidad de género en la empresa ha adquirido gran relevancia por su papel decisivo en las actividades innovadoras (Díaz-García et al., 2013; FernándezSastre, 2015; Jennings \& Brush, 2013). Se identifican dos líneas de investigación relacionadas con este tema. La primera, más consolidada, relaciona la diversidad de género y el rendimiento empresarial, demostrando que la paridad de hombres y mujeres dentro de la empresa promueve la maximización del rendimiento empresarial (Frink et al., 2003; Jehn et al., 1999; Williams \& O’Reilly, 1998), ya que provee al equipo de recursos cognitivos diversos relevantes para la gestión y el proceso de toma de decisiones (Hambrick, Cho, \& Chen, 1996).

La segunda línea, más emergente, estudia la diversidad de género y la innovación. La innovación es un cambio que supone una novedad. De acuerdo con el "Manual de Oslo" de la OCDE (Organización para la Cooperación y el Desarrollo Económico) del año 2005, por “innovación” se entiende la implementación con éxito de un producto o proceso nuevo (innovación radical), significativamente mejorado (innovación incremental) en el mercado o en una empresa, o la implementación de cambios organizativos o de marketing en la empresa. De entre estos diferentes cambios que implica la innovación, en este trabajo centramos la atención en la innovación de producto por su mayor impacto en el éxito comercial. La innovación de producto consiste en el desarrollo de nuevos bienes o servicios, con la finalidad de introducirlos en el mercado para satisfacer una necesidad de un usuario externo (Lager, 2002; Wan, Ong, \& Lee, 2005). Asimismo, ésta puede diferenciarse de acuerdo al grado de novedad; es por ello que se puede clasificar en radical e incremental (Kessler \& Chakrabarti, 1999). La innovación radical es aquella que conlleva cambios drásticos de novedad para el mercado y la incremental se refiere a innovaciones de mejoramiento para la empresa (Garcia \& Calantone, 2002).

La diversidad de género incrementa la habilidad de la empresa para explotar conocimientos internos y externos, y cuanto mayor es dicha diversidad mayor será la probabilidad de combinar nuevos conocimientos (Vegt \& Janssen, 2003). Así se ha demostrado empíricamente que la diversidad en los equipos facilita el intercambio de información, la diversidad de ideas y perspectivas, la combinación de diferentes conocimientos y experiencias, la identificación de oportunidades innovadoras y el desarrollo de nuevos productos y servicios en la empresa (Alexiev et al., 2010; Miller \& Triana, 2009; Torchia, Calabrò, \& Huse, 2011).

Ahora bien, la mayoría de los trabajos anteriores se han centrado sólo en los equipos de alta dirección, quedando pendiente el estudio en el resto de la empresa. Excepcionalmente cabe citar el trabajo de Østergaard et al. (2011), que encontró una fuerte relación positiva entre la diversidad de género y la innovación a nivel de empresa y que a su vez concluyeron que las empresas con una composición equilibrada de hombres y mujeres tienen más probabilidad de innovar en comparación con aquellas que tienen una alta concentración de un solo género. Además, el trabajo de Fernández-Sastre (2015), en una muestra de empresas manufactureras españolas, confirma el efecto positivo de la diversidad de género en la innovación, especialmente en la de producto, en equipos de I+D. También el trabajo reciente de Díaz-García et al. (2013) demuestra empíricamente, en una muestra de empresas españolas, una relación positiva entre la diversidad de género en el personal de I+D y la innovación radical. Por lo tanto, a partir de la revisión de la literatura y la necesidad de avanzar en el estudio de esta relación en el personal de I+D planteamos la siguiente hipótesis:

H1: La diversidad de género de empleados de I+D favorece la innovación de producto.

Por otro lado, los conocimientos, experiencias y habilidades de los empleados hacen que las empresas estén más abiertas a nuevas ideas y desarrollen una mayor capacidad 
de absorción para explotar el conocimiento externo e interno (Vegt \& Janssen, 2003). Así, los empleados con altos niveles educativos tienen una mayor habilidad para generar e implantar soluciones creativas e innovadoras (Bantel \& Jackson, 1989; Kimberly \& Evanisko, 1981; Oldham \& Cummings, 1996). Un nivel educativo alto, especialmente terciario, influye en la innovación, sobre todo en la de producto (McGuirk, Lenihan, \& Hart, 2015; Roach \& Sauermann, 2010), ya que son los que más contribuyen a la generación de nuevo conocimiento (Sauermann \& Cohen, 2008; Stephan, 2006). Ahora bien, al igual que en la diversidad de género, en este caso muchas investigaciones también se han centrado sólo en analizar el efecto de la educación en la innovación en los equipos de alta dirección (Heavey \& Simsek, 2013; Phan et al., 2009).

Por lo tanto, a partir de la revisión de la literatura que muestra una relación positiva entre el nivel educativo y la innovación (Alexiev et al., 2010; Heavey \& Simsek, 2013; Marvel \& Lumpkin, 2007; Wu et al., 2007) y específicamente de aquellos trabajos que demuestran que la alta formación de los empleados permite a las empresas estar más abiertas a nuevas ideas y a la búsqueda de soluciones creativas que propician la innovación (Alexiev et al., 2010; Deakins, Glancey, Menter, \& Wyper, 2005; Heavey \& Simsek, 2013; Honig, 2001; Unger, Rauch, Frese, \& Rosenbusch, 2011), planteamos la siguiente hipótesis:

H2: El alto nivel educativo de los empleados de I+D favorece la innovación de producto.

En los últimos 30 años el número de mujeres con educación universitaria ha aumentado progresivamente y en un gran número de países las mujeres estudian, en la actualidad y desde las últimas décadas, carreras que tradicionalmente se consideraban masculinas, tales como las ingenierías y la dirección de empresas. Además, hoy en día encontramos mujeres, especialmente en cargos de responsabilidad, que adoptan estereotipos masculinos que se relacionan con comportamientos innovadores (Gupta, Turban, Wasti, \& Sikdar, 2009). Estos hechos junto con la fuerte incorporación de la mujer en el mercado laboral han propiciado la heterogeneidad de género con alto nivel de formación en las empresas. Por lo tanto, esta realidad hace que resulte interesante estudiar el efecto moderador que la educación puede ejercer en la relación positiva existente entre la diversidad de género y la innovación.

Así, el hecho de que los empleados y empleadas tengan un mayor nivel formativo o educativo permite a las empresas estar más abiertas a nuevas ideas y a la búsqueda de soluciones creativas a los problemas (Fuentes, Bojica, \& Arroyo, 2010; Kimberly \& Evanisko, 1981; Vegt \& Janssen, 2003). Cabe esperar entonces que la relación diversidad de género e innovación se fortalezca a través de la educación o nivel formativo de los empleados, ya que se ha demostrado que el nivel educativo potencia el desempeño laboral y el desarrollo de ideas innovadoras (Deakins et al., 2005; Heavey \& Simsek, 2013; Unger et al., 2011), si se compara con empleados con bajo nivel formativo (Ployhart \& Moliterno, 2011). Así, el mayor nivel formativo o educativo existente en las empresas y que se corresponde en gran medida con la inserción de mujeres altamente cualificadas en el mundo laboral potenciará la relación entre la diversidad de género y la innovación. Este hecho puede cobrar mayor relevancia aún en los empleados de $I+D$, ya que estos empleados son los responsables de la planificación, desarrollo y coordinación de muchas de las innovaciones y gran parte de las patentes que desarrolla la empresa (Sauermann \& Cohen, 2008). Además, muchos de estos empleados son los que tienen un mayor vínculo con la comunidad científica, y por lo general pertenecen a asociaciones profesionales y asisten frecuentemente a reuniones científicas.

De este modo, se pretende contrastar si la innovación se puede beneficiar en mayor medida de la heterogeneidad de género de los empleados de I+D si estos poseen un alto nivel educativo, que les proporcione los conocimientos, habilidades y experiencias necesarias para descubrir, evaluar y explotar en mayor medida nuevas oportunidades creando nuevos productos (Shane \& Venkataraman, 2000). Por ello, planteamos la siguiente hipótesis:

H3: La influencia positiva de la diversidad de género en la innovación de producto aumentará con el alto nivel educativo en los empleados de I+D.

\section{METODOLOGÍA}

\section{Población y muestra}

Para contrastar la influencia de las variables seleccionadas en este estudio hemos utilizado como fuente de información la encuesta de innovación tecnológica en España, que pertenece al plan general de estadísticas de ciencia y tecnología de la Unión Europea (Eurostat). Para este estudio se han tenido en cuenta los datos de 2011 y 2012. Respecto a la selección de la muestra, de las 12.838 empresas que forman el panel en 2012 se tuvieron en cuenta dos criterios de selección: que las empresas se encontraran operativas y sin incidencias en los dos años, y que tuvieran siempre más de 10 empleados. Así, la muestra final está formada por un total de 7.962 empresas, de las cuales el $35 \%$ tiene menos de 20 años de antigüedad y el $65 \%$ más de 20 años. En cuanto al tamaño, se observa que el $76 \%$ son empresas 
pequeñas; el 12\%, medianas; y el $12 \%$, grandes empresas. En relación a la composición sectorial de la muestra, se observa que el $59 \%$ de la muestra son empresas manufactureras y el $41 \%$, de servicios.

\section{Medición de las variables}

\section{Variable dependiente}

La innovación de producto se ha medido distinguiendo entre innovación de bienes e innovación de servicios, así como mediante la innovación radical e incremental, según el grado de novedad de la misma. De acuerdo a las variables recogidas en la encuesta de innovación tecnológica y las medidas de innovación utilizadas en estudios previos (Simonen \& McCann, 2008), hemos empleado variables dicotómicas para cada uno de los tipos de innovación, que toman valor 1 si la empresa ha innovado.

\section{Variables independientes}

Para medir la diversidad de género de los empleados de I+D hemos empleado el índice de Blau. Éste es considerado como el índice más fiable y consistente de heterogeneidad (Bantel \& Jackson, 1989; Díaz-García et al., 2013; McMillan-Capehart \& Simerly, 2008). Se calcula como $B=1-\sum_{i=1}^{k}(p i)^{2}$, donde $B$ es el índice de Blau y $p$ es el porcentaje de miembros en cada i-ésima categoría de las k existentes (en este caso una para cada sexo, luego $\mathrm{K}=2$ ). Cuanto más elevado es el valor de $B$, mayor será el grado de diversidad, y dado que los valores varían entre o y (k-1)/k, la diversidad máxima será cuando $B$ alcance el valor de 0,5. Para calcular la diversidad de género mediante este índice se ha tomado el porcentaje de hombres y mujeres dedicados a las actividades de I+D interna. Para medir el alto nivel de educación de los empleados hemos utilizado el nivel educativo alcanzado por los empleados de I+D. Siguiendo la propuesta de Bantel y Jackson (1989), el nivel educativo se clasifica en categorías fijas que van desde la educación primaria hasta los estudios de doctorado. Así, se calculó el alto nivel de educación de los empleados teniendo en cuenta el porcentaje total de empleados de I+D con nivel de estudios de doctores, licenciados y diplomados. Finalmente, para el análisis del efecto moderador del nivel de educación en la relación entre la diversidad de género y la innovación se ha obtenido un término de interacción entre el indicador de diversidad de género en I+D multiplicado por el indicador de empleados con alto nivel de educación en I+D.

\section{Variables de control}

Adicionalmente, se incluyeron cinco variables de control que pudieran tener algún efecto sobre la innovación: el tamaño y antigüedad de la empresa, así como el sector y la cuantía dedicada a gastos en I+D internos y externos (Acs \& Preston, 1997; Galende \& Fuente, 2003; Orser, Riding, \& Manley, 2006; Zahra, Neubaum, \& Huse, 2000). El tamaño de la empresa se ha medido como el logaritmo neperiano del número de empleados del año 2012 (Cardinal, 2001; Pérez-Luño, Cabello-Medina, Carmona-Lavado, \& Cuevas-Rodríguez, 2011). La antigüedad de la empresa se ha medido con el número de años que tiene la empresa desde su constitución (Pérez-Luño et al., 2011) hasta el año 2012. Para medir el sector se ha creado una variable dicotómica que toma valor 1 cuando la empresa se dedica a actividades de servicios. Por último, los gastos de innovación se han medido con el porcentaje total de gastos internos y externos en I+D del año 2011.

\section{RESULTADOS}

Los análisis iniciales de los estadísticos descriptivos de las 7.962 empresas permitieron observar las siguientes frecuencias para los diferentes tipos de innovación en el total de la muestra. Así, el 39,6\% realiza innovación de producto; el 33\%, innovación de bienes; el 16,6\%, innovación de servicios; el 22,8\%, innovación radical; y el 30,6\%, innovación incremental. De acuerdo a los datos de estas frecuencias y, tal y como se indicará a continuación, dado el carácter dicotómico de estos indicadores, para el contraste de las hipótesis hemos considerado oportuna la obtención de muestras balanceadas que permitan dar mayor robustez a los modelos de regresión a calcular. Por ello, la Tabla 1 recoge directamente los estadísticos descriptivos, la colinealidad y las correlaciones de cada una de las submuestras balanceadas resultantes. En todas ellas se observa que las variables de innovación de producto, innovación de bien, innovación de servicio e innovación radical se correlacionan significativamente con las variables independientes.

A continuación, para el contraste de las hipótesis se procedió a utilizar la regresión logística binomial, siguiendo el método Wald hacia atrás. Para ello, se estimaron cinco modelos de regresión, uno para cada indicador de la variable dependiente. La tabla 2 recoge los resultados del último paso (método de Wald) de los cinco modelos de regresión logística binomial para la innovación de producto, bienes, servicios, radical e incremental. 
Tabla 1. Medias, desviaciones típicas, estadísticos de colinealidad y coeficientes de correlación de Pearson

\begin{tabular}{|c|c|c|c|c|c|c|c|c|c|c|c|c|c|}
\hline & \multirow[b]{2}{*}{ Media } & \multirow[b]{2}{*}{$\begin{array}{l}\text { Desv. } \\
\text { típica }\end{array}$} & \multirow[b]{2}{*}{ Frecuencias } & \multirow[b]{2}{*}{ FIV } & \multicolumn{9}{|c|}{ Correlaciones } \\
\hline & & & & & $\begin{array}{l}\text { Diversidad } \\
\text { género I+D }\end{array}$ & $\begin{array}{c}\text { Alto nivel } \\
\text { educaciónl+D }\end{array}$ & $\begin{array}{c}\text { Efecto } \\
\text { moderador }\end{array}$ & Tamaño & Antigüedad & Sector & $\begin{array}{c}\text { Gastos } \\
\text { internos I+D }\end{array}$ & $\begin{array}{c}\text { Gastos } \\
\text { externos I+D }\end{array}$ & Innovación \\
\hline \multicolumn{14}{|c|}{ Innovación de producto $-\mathrm{N}=6310$} \\
\hline Diversidad género en I+D & 0,1354 & 0,19173 & & 3,043 & 1 & & & & & & & & \\
\hline Alto nivel de educación en I+D & 25,24 & 33,604 & & 1,979 & $0,299^{\star \star}$ & 1 & & & & & & & \\
\hline Efecto moderador & 5,3355 & 10,97256 & & 3,205 & $0,688^{* *}$ & $0,629^{\star \star}$ & 1 & & & & & & \\
\hline Tamaño & 4,4863 & 1,45760 & & 1,136 & $0,089^{\star \star}$ & 0,019 & $0,125^{\star \star}$ & 1 & & & & & \\
\hline Antigüedad & 30,26 & 20,186 & & 1,098 & 0,013 & $0,026^{*}$ & $0,047^{\star \star}$ & $0,241^{\star \star}$ & 1 & & & & \\
\hline Sector & & & $1=2485 / 0=3825$ & 1,091 & $-0,057^{\star \star}$ & $-0,063^{\star \star}$ & $-0,038^{\star \star}$ & $0,152^{\star \star}$ & $-0,134^{\star \star}$ & 1 & & & \\
\hline Gastos internos en I+D & 40.78 & 43.350 & & 1,966 & $0,632^{\star \star}$ & $0,379^{\star \star}$ & $0,434^{\star \star}$ & $-0,034^{\star \star}$ & 0,000 & $-0,156^{\star \star}$ & 1 & & \\
\hline Gastos externos en I+D & 7.36 & 19.236 & & 1,046 & $0,093^{\star \star}$ & $0,057^{\star \star}$ & $0,079^{\star \star}$ & $0,078^{\star \star}$ & $0,038^{\star \star}$ & $-0,058^{\star \star}$ & $-0,050^{\star \star}$ & 1 & \\
\hline Innovación & & & $1=31550=3155$ & & $0,381^{* \star}$ & $0,245^{\star *}$ & $0,280^{\star \star}$ & $0,046^{* \star}$ & $0,031^{*}$ & $-0,140^{\star \star}$ & $0,464^{\star \star}$ & $0,124^{\star \star}$ & 1 \\
\hline \multicolumn{14}{|c|}{ Innovación de bienes $-\mathrm{N}=\mathbf{5 2 6 0}$} \\
\hline Diversidad género en I+D & 0,1460 & 0,19468 & & 3,013 & 1 & & & & & & & & \\
\hline Alto nivel de educación en I+D & 26,67 & 33,992 & & 2,030 & $0,295^{\star \star}$ & 1 & & & & & & & \\
\hline Efecto moderador & 5,8386 & 11,34961 & & 3,299 & $0,687^{\star \star}$ & $0,638^{\star \star}$ & 1 & & & & & & \\
\hline Tamaño & 4,4892 & 1,45217 & & 1,136 & $0,098^{\star *}$ & $0,031^{\star}$ & $0,133^{\star \star}$ & 1 & & & & & \\
\hline Antigüedad & 30,53 & 20,538 & & 1,100 & 0,007 & 0,023 & $0,039^{\star \star}$ & $0,248 * \star$ & 1 & & & & \\
\hline Sector & & & $1=1936 / 0=3324$ & 1,081 & $-0,052^{\star \star}$ & $-0,053^{\star \star}$ & $-0,029^{\star}$ & $0,132^{\star *}$ & $-0,133^{\star \star}$ & 1 & & & \\
\hline Gastos internos en I+D & 43.62 & 43.258 & & 1,915 & $0,618^{\star \star}$ & $0,369^{\star \star}$ & $0,423^{\star \star}$ & $-0,038^{\star \star}$ & -0.004 & $-0,152^{\star \star}$ & 1 & & \\
\hline Gastos externos en I+D & 7.78 & 19.466 & & 1,048 & $0,081^{\star \star}$ & $0,050^{\star \star}$ & $0,067^{\star \star}$ & $0,083^{\star \star}$ & $0,037^{\star \star}$ & $-0,056^{\star *}$ & $-0,069^{\star \star}$ & 1 & \\
\hline Innovación & & & $1=2630 / 0=2630$ & & $0,345^{\star \star}$ & $0,242^{\star \star}$ & $0,260^{\star \star}$ & 0,015 & $0,039^{\star \star}$ & $-0,277^{\star \star}$ & $0,446^{\star \star}$ & $0,117^{\star \star}$ & 1 \\
\hline \multicolumn{14}{|c|}{ Innovación de servicios - N = 2646} \\
\hline Diversidad género en I+D & 0,1690 & 0,19998 & & 2,943 & 1 & & & & & & & & \\
\hline Alto nivel de educación en I+D & 28,56 & 34,538 & & 2,242 & $0,280^{\star \star}$ & 1 & & & & & & & \\
\hline Efecto moderador & 6,7492 & 11,85531 & & 3,449 & $0,663^{\star *}$ & $0,667^{\star \star}$ & 1 & & & & & & \\
\hline Tamaño & 4,6075 & 1,55339 & & 1,161 & $0,079^{\star *}$ & $0,045^{*}$ & $0,135^{\star \star}$ & 1 & & & & & \\
\hline Antigüedad & 30,41 & 21,635 & & 1,129 & -0.01 & 0,030 & $0,040^{*}$ & $0,290^{\star \star}$ & 1 & & & & \\
\hline Sector & & & $1=1196 / 0=1450$ & 1,064 & 0,001 & -0.025 & -0.002 & $0,112^{\star \star}$ & $-0,132^{\star \star}$ & 1 & & & \\
\hline Gastos internos en I+D & 47.94 & 42.750 & & 1,932 & $0,612^{\star \star}$ & $0,367^{\star \star}$ & $0,414^{\star \star}$ & $-0,064^{\star *}$ & $-0,041^{\star}$ & $-0,099^{\star \star}$ & 1 & & \\
\hline Gastos externos en I+D & 8.38 & 19.466 & & 1,057 & $0,069^{\star \star}$ & $0,054^{\star \star}$ & $0,059^{\star \star}$ & $0,087^{\star \star}$ & 0,026 & $-0,039$ & $-0,097^{\star \star}$ & 1 & \\
\hline Innovación & & & $1=1323 / 0=1323$ & & $0,264^{\star \star}$ & $0,157^{\star \star}$ & $0,204^{\star \star}$ & $0,131^{\star \star}$ & -0.016 & $0,226^{\star \star}$ & $0,273^{\star \star}$ & 0,031 & 1 \\
\hline \multicolumn{14}{|c|}{ Innovación radical - N = 2686} \\
\hline Diversidad género en I+D & 0,2048 & 0,20209 & & 2,607 & 1 & & & & & & & & \\
\hline Alto nivel de educación en I+D & 33,59 & 34,918 & & 2,328 & $0,198^{* *}$ & 1 & & & & & & & \\
\hline Efecto moderador & 8,2637 & 12,64734 & & 3,610 & $0,640^{\star \star}$ & $0,661^{\star \star}$ & 1 & & & & & & \\
\hline Tamaño & 4,5588 & 1,45612 & & 1,191 & $0,108^{\star \star}$ & $0,075^{\star \star}$ & $0,165^{\star \star}$ & 1 & & & & & \\
\hline Antigüedad & 30,88 & 20,266 & & 1,165 & 0,013 & $0,054^{\star \star}$ & $0,059^{\star \star}$ & $0,323^{\star \star}$ & 1 & & & & \\
\hline Sector & & & $1=870 / 0=1816$ & 1,064 & $0,042^{*}$ & -0.009 & $0,042^{*}$ & $0,055^{\star \star}$ & $-0,171^{\star \star}$ & 1 & & & \\
\hline Gastos internos en I+D & 60.15 & 39.400 & & 1,629 & $0,478^{\star \star}$ & $0,288^{\star \star}$ & $0,311^{\star *}$ & $-0,096^{\star \star}$ & -0.013 & $-0,088^{\star \star}$ & 1 & & \\
\hline Gastos externos en I+D & 9.83 & 20.501 & & 1,102 & -0.009 & -0.009 & 0,008 & 0,090 & $0,028^{\star \star}$ & -0.024 & $-0,257^{\star \star}$ & 1 & \\
\hline Innovación & & & $1=1343 / 0=1343$ & & $0,133^{\star \star}$ & $0,132^{\star \star}$ & $0,138^{\star *}$ & 0,059 & $0,016^{\star \star}$ & $0,040^{*}$ & $0,133^{\star \star}$ & -0.016 & 1 \\
\hline \multicolumn{14}{|c|}{ Innovación incremental - N = 1436} \\
\hline Diversidad género en I+D & 0,2119 & 0,20304 & & 2,629 & 1 & & & & & & & & \\
\hline Alto nivel de educación en I+D & 33,67 & 34,886 & & 2,441 & $0,193^{\star \star}$ & 1 & & & & & & & \\
\hline Efecto moderador & 8,4883 & 12,95063 & & 3,720 & $0,631^{\star \star}$ & $0,670^{\star \star}$ & 1 & & & & & & \\
\hline Tamaño & 4,5032 & 1,50437 & & 1,187 & $0,071^{\star \star}$ & $0,079^{\star \star}$ & $0,146^{\star \star}$ & 1 & & & & & \\
\hline Antigüedad & 29,96 & 19,671 & & 1,210 & 0,020 & 0,037 & $0,058^{*}$ & $0,344^{\star \star}$ & 1 & & & & \\
\hline Sector & & & $1=905 / 0=531$ & 1,093 & $0,067^{\star}$ & -0.001 & 0,030 & 0,014 & $-0,23^{\star *}$ & 1 & & & \\
\hline Gastos internos en I+D & 61.84 & 38.719 & & 1,633 & $0,458^{\star \star}$ & $0,282^{\star \star}$ & $0,279^{\star *}$ & $-0,091^{\star *}$ & -0.013 & $-0,075^{\star *}$ & 1 & & \\
\hline Gastos externos en I+D & 10.08 & 20.042 & & 1,118 & 0,021 & 0,004 & 0,043 & $0,077^{\star \star}$ & 0,038 & -0.023 & $-0,263^{\star}$ & 1 & \\
\hline Innovación & & & $1=718 / 0=718$ & & -0.013 & -0.009 & -0.001 & $0,104^{\star \star}$ & $0,073^{\star \star}$ & $-0,065^{*}$ & -0.012 & 0,003 & 1 \\
\hline
\end{tabular}

Nota: *La Correlación es significativa al nivel o,05 (bilateral) ** La correlación es significativa al nivel o,01 (bilateral) 
Tabla 2. Regresiones de la variable dependiente

\begin{tabular}{|c|c|c|c|c|c|c|c|c|c|c|c|c|c|c|c|c|c|c|c|c|}
\hline \multirow{2}{*}{ Innovación } & \multicolumn{4}{|c|}{$\begin{array}{c}\text { Modelo } 1 . \\
\text { Innovación producto }\end{array}$} & \multicolumn{4}{|c|}{$\begin{array}{c}\text { Modelo } 2 . \\
\text { Innovación bienes }\end{array}$} & \multicolumn{4}{|c|}{$\begin{array}{c}\text { Modelo } 3 . \\
\text { Innovación servicios }\end{array}$} & \multicolumn{4}{|c|}{$\begin{array}{c}\text { Modelo } 4 . \\
\text { Innovación radical }\end{array}$} & \multicolumn{4}{|c|}{$\begin{array}{c}\text { Modelo } 5 . \\
\text { Innovación incremental }\end{array}$} \\
\hline & B & Wald & Sig & $\operatorname{Exp}(B)$ & B & Wald & Sig & $\operatorname{Exp}(B)$ & B & Wald & Sig & $\operatorname{Exp}(B)$ & B & Wald & Sig & $\operatorname{Exp}(B)$ & B & Wald & Sig & $\operatorname{Exp}(B)$ \\
\hline $\begin{array}{l}\text { Diversidad de } \\
\text { género I+D }\end{array}$ & 1,461 & 54,843 & 0,000 & 4,309 & 1,194 & 32,052 & 0,000 & 3,300 & 1,095 & 16,466 & 0,000 & 2,988 & 0,689 & 9,429 & 0,002 & 1,992 & - & - & - & - \\
\hline $\begin{array}{l}\text { Alto nivel de } \\
\text { Educación I+D }\end{array}$ & 0,005 & 24,813 & 0,000 & 1,005 & 0,006 & 29,686 & 0,000 & 1,006 & 0,003 & 4,918 & 0,027 & 1,003 & 0,005 & 20,676 & 0,000 & 1,005 & - & - & - & - \\
\hline $\begin{array}{l}\text { Diversidadgénerol } \\
+D^{\star} \text { Alto Nivel } \\
\text { Educaciónl + D } \\
\end{array}$ & - & - & - & - & - & - & - & - & - & - & - & - & - & - & $\cdot$ & $\cdot$ & - & - & - & $\cdot$ \\
\hline Constante & -1.790 & 251,602 & 0,000 & 0,167 & -2.239 & 305,360 & 0,000 & 0,107 & -1.060 & 44,599 & 0,000 & 0,346 & -0.781 & 23,674 & 0,000 & 0,458 & -0.813 & 19,402 & 0,000 & 0,443 \\
\hline Tamaño & 0,088 & 18,308 & 0,000 & 1,092 & 0,060 & 6,672 & 0,010 & 1,061 & 0,165 & 33,266 & 0,000 & 1,179 & 0,072 & 6,642 & 0,010 & 1,074 & 0,142 & 15,708 & 0,000 & 1,152 \\
\hline Antigüedad & - & - & - & $\cdot$ & - & - & - & - & - & - & - & - & - & - & - & - & - & - & - & - \\
\hline Sector & 0,393 & 41,394 & 0,000 & 1,482 & 1,173 & 286,159 & 0,000 & 3,231 & -1.140 & 161,501 & 0,000 & 0,320 & -0.189 & 4,947 & 0,026 & 0,828 & 0,278 & 6,346 & 0,012 & 1,321 \\
\hline $\begin{array}{l}\text { Gastos innovación } \\
\text { internos I+D }\end{array}$ & 0,018 & 438,628 & 0,000 & 1,019 & 0,018 & 345,308 & 0,000 & 1,018 & 0,013 & 87,512 & 0,000 & 1,013 & 0,004 & 13,058 & 0,000 & 1,004 & - & - & - & - \\
\hline $\begin{array}{l}\text { Gastos innovación } \\
\text { externos I+D }\end{array}$ & 0,013 & 88,944 & 0,000 & 1,013 & 0,013 & 72,824 & 0,000 & 1,013 & .005 & 6.079 & 0,014 & 1,005 & $\cdot$ & - & - & - & - & - & - & - \\
\hline -2 Log Verosimilitud & \multicolumn{4}{|c|}{7036,686} & \multicolumn{4}{|c|}{5719,574} & \multicolumn{4}{|c|}{3196,425} & \multicolumn{4}{|c|}{3623,588} & \multicolumn{4}{|c|}{1968,679} \\
\hline $\mathrm{R}^{2}$ Cox-Snell & \multicolumn{4}{|c|}{0,237} & \multicolumn{4}{|c|}{0,258} & \multicolumn{4}{|c|}{0,163} & \multicolumn{4}{|c|}{0,037} & \multicolumn{4}{|c|}{0,015} \\
\hline $\mathrm{R}^{2}$ Nagelkerke & \multicolumn{4}{|c|}{0,317} & \multicolumn{4}{|c|}{0,345} & \multicolumn{4}{|c|}{0,218} & \multicolumn{4}{|c|}{0,049} & \multicolumn{4}{|c|}{0,020} \\
\hline$X^{2}$ & \multicolumn{4}{|c|}{1710.831} & \multicolumn{4}{|c|}{1572.335} & \multicolumn{4}{|c|}{471,710} & \multicolumn{4}{|c|}{99.998} & \multicolumn{4}{|c|}{22.040} \\
\hline Significación & \multicolumn{4}{|c|}{0,000} & \multicolumn{4}{|c|}{0,000} & \multicolumn{4}{|c|}{0,000} & \multicolumn{4}{|c|}{0,000} & & & & \\
\hline $\mathrm{N}$ & & 631 & & & & 52 & & & & 26 & & & & 26 & & & & 14 & & \\
\hline $\begin{array}{l}\text { \% Global Tabla } \\
\text { clasificación }\end{array}$ & & 75 & & & & 74 & & & & 66 & & & & 58 & & & & 57 & & \\
\hline
\end{tabular}

Nota: Punto de corte para la clasificación 0,5

Respecto a la hipótesis 1, que proponía la influencia positiva de la diversidad de género en los equipos de I+D en la innovación de producto, los resultados de los modelos para la innovación de producto, innovación de bien, innovación de servicio e innovación radical permiten concluir que esta hipótesis se confirma de forma positiva y significativa. Así, los resultados de los modelos 1; 2; 3 y 4 muestran que el coeficiente de regresión es siempre positivo y significativo, lo que indica que la diversidad de género del personal dedicado a las actividades de I+D hace más probable la innovación 4,309 veces para la innovación de producto; 3,300 veces para la innovación de bienes; 2,988 veces para la innovación de servicios; y 1,992 veces para la innovación radical.

Respecto a la hipótesis 2, que proponía una influencia positiva del nivel de educación de los empleados dedicados a las actividades de I+D en la innovación de producto, los resultados de los modelos 1; 2; 3 y 4 de regresión permiten confirmar que un alto nivel de educación de los empleados de I+D favorece positiva y significativamente la innovación producto, lo que indica que el alto nivel de educación de los empleados de I+D hace más probable la innovación en particular 1,005 veces para la innovación de producto; 1,006 veces para la innovación de bien; 1,003 veces para la innovación de servicios; y 1.005 veces para la innovación radical.

Finalmente, respecto a la hipótesis 3 , que establecía que la influencia positiva de la diversidad de género del personal de I+D en la innovación de producto aumentaría con el alto nivel de educación de los empleados de I+D, los resultados obtenidos no han permitido confirmar esta hipótesis. Se observa que el efecto interacción entre la diversidad de género y alto nivel educativo de los empleados en I+D no tienen relación significativa para ningún indicador de la innovación de producto.

En relación a las variables de control, con respecto a la innovación de productos, innovación de bien, innovación de servicio, innovación radical e incremental como variables dicotómicas dependientes, los modelos indican que la variable antigüedad no tiene relación significativa. En cuanto a la variable gastos de innovación externos en $\mathrm{I}+\mathrm{D}$, los modelos indican que esta no tiene relación significativa para la innovación radical e incremental. Asimismo, para la innovación incremental los gastos de innovación internos en I+D no tienen relación significativa. Las variables de control sector y tamaño son positivas y significativas para todos los indicadores de la variable dependiente.

\section{DISCUSIÓN Y CONCLUSIONES}

La innovación es clave para la renovación de la empresa, el éxito empresarial y juega un papel crucial en tiempos de crisis; y la innovación de producto, especialmente, es un elemento vital para competir en los entornos cada vez más dinámicos (Hoskisson, Covin, Volberda, \& Johnson, 2011; Slater, Mohr, \& Sengupta, 2014). Aunque es muy abundante la literatura que analiza los factores 
que influyen en la innovación, hay dos factores, la diversidad de género y el nivel de educación, cuyo impacto en la innovación no ha sido suficientemente estudiado, especialmente en los empleados de I+D. Por ello, este trabajo ha pretendido avanzar y aportar nueva evidencia al estudio de la influencia de la diversidad de género y el nivel educativo en la innovación.

Tomando en cuenta aquellas investigaciones que ponen de manifiesto la influencia positiva de la diversidad de género y el nivel educativo en la innovación (Alexiev et al., 2010; Østergaard et al., 2011), realizamos 5 modelos de regresión logística binomial con los datos extraídos de la encuesta de innovación tecnológica en España, para demostrar el efecto de estas relaciones en el caso de los empleados de I+D.

Los resultados de nuestro trabajo han demostrado que en los empleados de I+D, la diversidad de género favorece la innovación de producto que resulta ser de novedad para el mercado. Estos hallazgos están en línea con el trabajo de Alexiev et al. (2010), que comprobó empíricamente que la diversidad en los equipos de dirección facilita el desarrollo de nuevos productos y servicios en la empresa. Y con el de Fernández-Sastre (2015), que concluyó que la diversidad de género en los equipos de I+D favorece la innovación, especialmente de producto. También, con los resultados de Østergaard et al. (2011), que confirmaron que la diversidad de género en los empleados tiene un efecto positivo en la probabilidad de que las empresas innoven. Así, se concluye que en los empleados de I+D esta diversidad también es importante para la innovación, ya que favorece el contraste de opiniones (García \& Moreno, 2012), permitiéndole a la empresa la posibilidad de combinar diferentes conocimientos y habilidades que promueven la creatividad y la innovación de producto. Asimismo, la confirmación de esta relación en nuestro trabajo proporciona nueva evidencia empírica para los equipos de I+D en el contexto español, lo que demuestra la importancia de la diversidad de género en la innovación radical de producto; y la gran relevancia de esta innovación para que las empresas incrementen la habilidad de crear nuevos negocios, mejoren el rendimiento empresarial y, por ende, la posibilidad de sobrevivir en tiempos de crisis (Slater et al., 2014). Igualmente, este resultado tiene implicaciones prácticas, tanto a nivel empresarial, como para los que toman decisiones a nivel gubernamental. Los resultados claramente indican la relevancia y necesidad de promover un mayor número de políticas gubernamentales de empleo que favorezcan la diversidad de empleados de ambos sexos en todos los niveles jerárquicos de la empresa. A pesar de que en España existe alguna regulación, como la Ley Orgánica 3/2007, de 22 marzo, para la igualdad efectiva de mujeres y hombres en las empresas, o el código de buen gobierno para las empresas cotizadas, con sus recomendaciones sobre la presencia de mujeres en los consejos de administración, la realidad muestra que se está aún lejos de cumplirse y que existe poco avance sobre la igualdad de oportunidades para las mujeres en la empresa (García \& Moreno, 2012). Así, para que las empresas puedan aprovechar la influencia positiva de la diversidad de género en la innovación, es necesario que los gobiernos promuevan más políticas gubernamentales que faciliten la conciliación de la vida profesional y personal para garantizar que especialmente las mujeres tengan la misma facilidad de incorporación plena al mundo laboral que los hombres y éstas estén libres de cualquier tipo de estereotipo de género (Rivero, 2008; Hoobler, Wayne, \& Lemmon, 2009).

De igual forma, nuestros resultados concluyen que la presencia de empleados con nivel educativo superior enriquece la innovación en el caso de los empleados de l+D, ya que las habilidades y experiencias profesionales contribuyen a la generación de nuevas ideas y a la innovación radical de productos. Estos resultados concuerdan con los obtenidos en trabajos empíricos previos que han puesto de manifiesto que el nivel educativo o formativo de los empleados afecta de forma positiva a las decisiones y tendencias innovadoras en la empresa (Bantel \& Jackson, 1989; Wiersema \& Bantel, 1992); y que un nivel formativo superior de los empleados junto con la existencia de profesiones heterogéneos hacen que las empresas tengan una mayor probabilidad de innovar (Østergaard et al., 2011). Este trabajo contribuye con nueva evidencia empírica sobre cómo el nivel de educación de los empleados favorece la innovación más allá del ámbito de los equipos de la alta dirección. De esta forma, los resultados muestran que los empleados de I+D mejor formados aportan conocimientos y experiencias que proveen a la empresa de innovaciones radicales de productos, necesarias para alcanzar los objetivos que persigue la empresa. Así, se espera que los conocimientos y habilidades que adquieren los empleados en su proceso de formación ayuden a mejorar sus habilidades para innovar. Además, este resultado tiene importantes implicaciones empresariales y gubernamentales en la medida en que al confirmarse el efecto positivo que tiene la formación de los trabajadores en la innovación, las empresas y gobiernos deberán favorecer y desarrollar programas educativos y de formación que fomenten la creatividad e innovación (Bae, Qian, Miao, \& Fiet, 2014).

De igual forma, es importante destacar que ambas relaciones se han confirmado para la innovación de producto en bienes y servicios. Como conclusión general, podemos mencionar que la diversidad de género y el alto nivel educativo potencian la innovación, ya que a través de ellos la empresa puede obtener una variedad de conocimientos, experiencias y habilidades que hace que se encuentre más abierta a nuevas ideas (Fuentes et al., 2010; Vegt \& Janssen, 2003). De esta forma, se facilita el desarrollo de innovaciones radicales de productos de importancia para la entrada a nuevos mercados y para sortear de mejor manera las consecuencias que acarrea la actual crisis económica. 
Ahora bien, en relación a la existencia de un efecto moderador del alto nivel educativo entre la diversidad de género y la innovación en los equipos de $1+D$, los resultados indican que esta relación no es significativa. En concreto, esto puede deberse a dos motivos. En primer lugar, los resultados muestran que, a pesar de que ambas variables independientes tienen efecto en la innovación, la influencia positiva de la diversidad de género sobre la innovación de producto es mucho mayor. Por lo tanto, esto podría explicar que el efecto moderador del alto nivel educativo no tenga ningún efecto potenciador en la relación diversidad de género e innovación. En segundo lugar, los resultados también muestran una baja correlación entre las variables independientes en todos los modelos; por lo que podría esperarse que estas variables no tengan efecto una sobre la otra. Por lo tanto, quizás cabría la posibilidad de considerar que la relación diversidad de género e innovación pueda estar moderada por otras variables, tales como la satisfacción de los empleados o la efectividad de la comunicación (Díaz-García et al., 2013). Además, puede influir el hecho de que los hombres perciban como una amenaza la entrada de las mujeres igual o mejor formadas que ellos y que adoptan estereotipos masculinos. Los prejuicios de género masculino podrían estar impidiendo que se aprovechen todos los beneficios que cabría esperar de la diversidad de género en la empresa (García \& Moreno, 2012, Robinson \& Dechant, 1997). Así, la llegada de mujeres con alta formación ha podido generar un clima hostil de trabajo que impide que fluya la comunicación y repercute negativamente en la innovación. Estos resultados van en la misma línea de estudios previos que han confirmado que cuando la organización potencia y recompensa el talento se encuentran importantes diferencias en la comparación entre hombres y mujeres, la denominada por Castilla y Benard (2010) "paradoja de la meritocracia". Por lo tanto, para que la relación positiva de la diversidad de género en la innovación se potencie, es necesario que las empresas y sus empleados estén libres de cualquier prejuicio de género. De esta forma, se desarrollarán nuevos paradigmas en los cuales lo más importante para la empresas sean los recursos humanos y la formación de los mismos, reconociendo que el talento se encuentra tanto en hombres como en mujeres y que es un perjuicio para las empresas su desaprovechamiento (Gallego \& Briones, 2007). Esto sin duda influirá en el ambiente laboral, propiciando las condiciones necesarias para que tanto hombres como mujeres potencien su talento y pongan en práctica sus ideas innovadoras.

Por todo ello, las principales aportaciones del trabajo son las siguientes: Por una parte, se ha podido demostrar que la diversidad de género y el alto nivel de educación de los empleados influyen positivamente en la innovación radical de productos, para el caso español, incluyendo tanto empresas del sector servicios como de manufactura y considerando como nivel de análisis el personal de I+D. Los estudios previos sólo analizan estas relaciones en los equipos de alta dirección y en muestras de otros países (Heavey \& Simsek, 2013; Østergaard et al., 2011). Además, los trabajos anteriores no suelen incluir empresas del sector servicios (Phan et al., 2009). Todo esto constituye una aportación tanto para la literatura de innovación como de recursos humanos. Por otra parte, los resultados evidencian que no es significativo el efecto potenciador del alto nivel formativo de los empleados en la relación diversidad de género e innovación. Esto puede deberse principalmente a que estas variables tienen baja correlación entre sí, por lo que podría esperarse que el efecto potenciador pueda venir en mayor medida por otras variables, como la satisfacción de los miembros del equipo y la efectividad de la comunicación. Por ello, a pesar de los resultados positivos de la diversidad de género y del alto nivel educativo de los empleados de I+D por separado en la innovación, es de importancia que las empresas y gobiernos promuevan más políticas gubernamentales que faciliten la conciliación de la vida profesional y personal de las mujeres, que éstas tengan la misma facilidad de incorporación plena al mundo laboral que los hombres y que estén libres de cualquier prejuicio de género. De este modo, las empresas pondrán en valor la formación de todos sus empleados y empleadas, lo que las ayudará a posicionarse mejor en el mercado, ser rentables y sobrevivir en tiempo de crisis.

En todo caso, los resultados están condicionados por la fuente de información empleada y la forma en que se ha aproximado la medida de las variables. Proponemos para investigaciones futuras profundizar con mayor evidencia empírica en la evaluación del efecto moderador de variables como la creatividad, la satisfacción de los miembros, la efectividad de la comunicación y la capacidad de aprendizaje en la relación diversidad de género e innovación, e incluso entrando en un mayor detalle de observación de acuerdo al sector de actividad de la empresa, así como realizar estudios longitudinales mediante la construcción de un panel con el mayor número de años posibles atendiendo a las variables a analizar. Asimismo, se pretende testar las hipótesis mencionadas en este trabajo en una muestra representativa de empresas de un país en vía de desarrollo y comparar los resultados. En estos países, a pesar de haberse producido una mejora en el nivel educativo, en especial de los jóvenes, se observa que siguen careciendo de la formación de más alto nivel que es la que más promueve la innovación. Además, en estos países las mujeres jóvenes son las que suelen tener mayor nivel formativo pero al mismo tiempo son las más afectadas por el paro juvenil, por lo que es muy poco habitual encontrar mujeres altamente cualificadas en el ámbito empresarial (Banco Mundial, 2013). 


\section{REFERENCIAS}

Acs, Z. J., \& Preston, L. (1997). Small and medium-sized enterprises, technology, and globalization: Introduction to a special issue on small and medium-sized enterprises in the global economy. Small Business Economics, 9(1), 1-6. doi:10.1023/a:1007945327618

Alexiev, A., Jansen, J., Bosch, A. Van den, \& Volberda, H. (2010). Top management team advice seeking and exploratory innovation: The moderating role of TMT heterogeneity. Journal of Management Studies, 47(7), 1343-1364. doi:10.1111/j.1467-6486.2010.00919.x

Bae, T. J., Qian, S., Miao, C., \& Fiet, J. O. (2014). The relationship between entrepreneurship education and entrepreneurial intentions: A metaAnalytic Review. Entrepreneurship Theory and Practice, 38(2), 217254. doi:10.1111/etap.12095

Banco Mundial. (2013). Informe anual. Instituto de Relaciones Internacionales (IRI).

Bantel, K., \& Jackson, S. (1989). Top management and innovations in banking: Does the composition of the top team make a difference? Strategic Management Journal, 10(S1), 107-124. doi:10.1002/ smj.4250100709

Bruin, A., Brush, C. G., \& Welter, F. (2007). Advancing a framework for coherent research on women's entrepreneurship. Entrepreneurship: Theory and Practice, 31(3), 323-339. doi:10.1111/j.15406520.2007.00176.x

Cardinal, L. B. (2001). Technological innovation in the pharmaceutical industry: The use of organizational control in managing research and development. Organization Science, 12(1), 19-36. doi:10.1287/ orsc.12.1.19.10119

Castilla, E. J., \& Benard, S. (2010). The paradox of meritocracy in organizations. Administrative Science Quarterly, 55(4), 543-576. doi:10.2189/asqu.2010.55.4.543

Cefis, E., \& Marsili, O. (2006). Survivor: The role of innovation in firms' survival. Research Policy, 35(5), 626-641. doi:10.1016/j. respol.2006.02.006

Corbett, A., Covin, J. G., O’Connor, G. C., \& Tucci, C. L. (2013). Corporate entrepreneurship: State of the art research and a future research agenda. Journal of Product Innovation Management, 30(5), 812-820. doi:10.1111/jpim.12031

Crawford, C. B., Gould, L. V., \& Scott, R. F. (2003). Transformational leader as champion and techie: Implications for leadership educators Journal of Leadership Education, 2(1), 1-12.

Deakins, D., Glancey, K., Menter, I., \& Wyper, J. (2005). Enterprise education: The role of head teachers. International Entrepreneurship and Management Journal, 1(2), 241-263. doi:10.1007/s11365-0051131-9

DeLong, D. W., \& Fahey, L. (2000). Diagnosing cultural barriers to knowledge management. Academy of Management Executive, 14(4), 113-127.

Díaz-García, C., González-Moreno, A., \& Sáez-Martínez, F. (2013). Gender diversity within R\&D teams: Its impact on radicalness of innovation. Innovation: Organization \& Management, 15(2), 149-160. doi:10.5172/impp.2013.15.2.149

Donate, M. J., \& Guadamillas, F. (2011). Organizational factors to support knowledge management and innovation. Journal of Knowledge Management, 15(6), 890-914. doi:10.1108/13673271111179271
Doorn, S. Van, Jansen, J. P., Bosch, F. A. J. Van den, \& Volberda, H. W. (2013). Entrepreneurial orientation and firm performance: Drawing attention to the senior team. Journal of Product Innovation Management, 30(5), 821-836. doi:10.1111/jpim.12032

Fernández-Sastre, J. (2015). The impact of R\&D teams' gender diversity on innovation outputs. International Journal of Entrepreneurship and Small Business, 24(1), 142-162. doi:10.1504/ijesb.2015.066154

Frink, D., Robinson, R., Reithel, B., Arthur, M., Ammeter, A., Ferris, G. ... Morrisette, H. (2003). Gender demography and organizational performance: A two study investigation with convergence. Group \& Organization Management, 28(1), 127-147. doi:10.1177/1059601102250025

Fuentes, M., Bojica, A., \& Arroyo, M. R. (2010). La orientación emprendedora en las iniciativas empresariales de mujeres: Influencia de las relaciones externas. Cuadernos de Estudios Empresariales, 20, 35-53.

Galende, J., \& Fuente, J. De la. (2003). Internal factors determining a firm's innovative behaviour. Research Policy, 32(5), 715-736. doi:10.1016/So048-7333(02)00082-3

Gallego, A. C., \& Briones, J. L. (2007). La diversidad de género en el Código Unificado español y la práctica empresarial. Pecvnia: Revista de la Facultad de Ciencias Económicas y Empresariales, 4, 1-25.

Garcia, R., \& Calantone, R. (2002). A critical look at technological innovation typology and innovativeness terminology: A literature review. Journal of Product Innovation Management, 19(2), 110-132. doi:10.1111/1540-5885.1920110

García, M. C. D., \& Moreno, A. G. (2012). La cuota de mujeres en los consejos de administración: ¿2015 una utopía? Boletín Económico de ICE, 3027, 53-61.

Gupta, V. K., Goktan, A. B., \& Gunay, G. (2014). Gender differences in evaluation of new business opportunity: A stereotype threat perspective. Journal of Business Venturing, 29(2), 273-288. doi:10.1016/j.jbusvent.2013.02.002

Gupta, V. K., Turban, D., Wasti, S. A., \& Sikdar, A. (2009). The role of gender stereotypes in perceptions of entrepreneurs and intentions to become an entrepreneur. Entrepreneurship Theory \& Practice, 33(2), 397-417. doi:10.1111/j.1540-6520.2009.00296.x

Hambrick, D., Cho, T., \& Chen, M. (1996). The influence of top management team heterogeneity on firms' competitive moves. Administrative Science Quarterly, 41(4), 659-684. doi:10.2307/2393871

Hayton, J. (2005). Competing in the new economy: The effect of intellectual capital on corporate entrepreneurship in high-technology new ventures. R\&D Management, 35(2), 137-155. doi:10.1111/j.14679310.2005.00379.x

Hayton, J. C., \& Kelley, D. J. (2006). A competency-based framework for promoting corporate entrepreneurship. Human Resource Management, 45(3), 407-427. doi:10.1002/hrm.20118

Heavey, C., \& Simsek, Z. (2013). Top management compositional effects on corporate entrepreneurship: The moderating role of perceived technological uncertainty. Journal of Product Innovation Management, 30(5), 837-855. doi:10.1111/jpim.12033

Honig, B. (2001). Learning strategies and resources for entrepreneurs and intrapreneurs. Entrepreneurship: Theory and Practice, 26(1), 21-35.

Hoobler, J. M., Wayne, S. J., \& Lemmon, G. (2009). Bosses' perceptions of family-work conflict and women's promotability: Glass ceiling effects. Academy of Management Journal, 52(5), 939-957. 
Horwitz, S. (2005). The compositional impact of team diversity on performance: Theoretical consideration. Human Resource Development Review, 4(2), 219-245. doi:10.1177/1534484305275847

Hoskisson, R. E., Covin, J., Volberda, H. W., \& Johnson, R. A. (2011). Revitalizing entrepreneurship: The search for new research opportunities. Journal of Management Studies, 48(6), 1141-1168. doi:10.1111/j.1467-6486.2010.00997.x

Jehn, K., Northcraft, G., \& Neale, M. (1999). Why differences make a difference: A field study of diversity, conflict, and performance in work groups. Administrative Science Quarterly, 44(4), 741-763. doi:10.2307/2667054

Jennings, J., \& Brush, C. (2013). Research on women entrepreneurs: Challenges to (and from) the broader entrepreneurship literature?. The Academy of Management Annals, 7(1), 661-715. doi:10.1080/19 416520.2013 .782190

Kearney, E., Gebert, D., \& Voelpel, S. C. (2009). When and how diversity benefits teams: The importance of team members' need for cognition. Academy of Management Journal, 52(3), 581-598. doi:10.5465/ amj.2009.41331431

Kessler, E. H., \& Chakrabarti, A. K. (1999). Speeding up the pace of new product development. Journal of Product Innovation Management, 16(3), 231-247. doi:10.1016/S0737-6782(98)00048-4

Kimberly, J. R., \& Evanisko, M. J. (1981). Organizational innovation: The Influence of individual, organizational, and contextual factors on hospital adoption of technological and administrative innovations. Academy of Management Journal, 24(4), 689-713. doi: $10.2307 / 256170$

Lager, T. (2002). Product and process development intensity in process industry: A conceptual and empirical analysis of the allocation of company resources for the development of process technology. International Journal of Innovation Management, 6(2), 105-130. doi:10.1142/S1363919602000537

Liu, N. C., \& Liu, M. S. (2011). Human resource practices and individual knowledge-sharing behavior- an empirical study for Taiwanese R\&D professionals. The International Journal of Human Resource Management, 22(4), 981-997. doi:10.1080/09585192.2011.555138

Love, J. H., \& Roper, S. (2015). SME innovation, exporting and growth: A review of existing evidence. International Small Business Journal, 33(1), 28-48. doi:10.1177/0266242614550190

Manolopoulos, D. (2006). What motivates R\&D professionals? Evidence from decentralized laboratories in Greece. The International Journal of Human Resource Management, 17(4), 616-647. doi:10.1080/09585190600581394

Marvel, M. R., \& Lumpkin, G. T. (2007). Technology entrepreneur's human capital and its effects on innovation radicalness. Entrepreneurship Theory and Practice, 31(6), 807-828. doi:10.1111/ j.1540-6520.2007.00209.x

McGuirk, H., Lenihan, H., \& Hart, M. (2015). Measuring impact of innovative human capital on small firms' propensity to innovate. Research Policy, 44(4), 965-976. doi:10.1016/j.respol.2014.11.008

McMillan-Capehart, A., \& Simerly, R. (2008). Effects of managerial racial and gender diversity on organizational performance: An empirical study international. Journal of Management, 25(3), 446-451.
Miller, T., \& Triana, M. C. (2009). Demographic diversity in the boardroom: Mediators of the board diversity-firm performance relationship. Journal of Management Studies, 46(5), 755-786. doi:10.1111/j.14676486.2009.00839.x

Oldham, G. R., \& Cummings, A. (1996). Employee creativity: Personal and contextual factors at work. Academy of Management Journal, 39(3), 607-634.

Orser, B., Riding, A., \& Manley, K. (2006). Women entrepreneurs and financial capital. Entrepreneurship Theory and Practice, 30(5), 643665. doi:10.1111/j.1540-6520.2006.00140.x

Østergaard, C., Timmermans, B., \& Kristinsson, K. (2011). Does a different view create something new? The effect of employee diversity on innovation. Research Policy, 40(3), 500-509. doi:10.1016/j. respol.2010.11.004

Pérez-Luño, A., Cabello-Medina, C., Carmona-Lavado, A., \& CuevasRodríguez, G. (2011). How social capital and knowledge affect innovation. Journal of Business Research, 64(12), 1369-1376. doi:10.1016/j.jbusres.2011.01.014

Phan, P., Wright, M., Ucbasaran, D., \& Tan, W. (2009). Corporate entrepreneurship: Current research and future directions. Journal of Business Venturing, 24(3), 197-205. doi:10.1016/j. jbusvent.2009.01.007

Ployhart, R. E., \& Moliterno, T. P. (2011). Emergence of the human capital resource: A multilevel model. Academy of Management Review, 36(1), 127-150. doi:10.5465/amr.2009.0318

Rivero, A. (2008). Conciliación de la vida familiar y la vida laboral: Situación actual, necesidades y demandas. Madrid, España: Instituto de la Mujer, M-34729-2005.

Roach, M., \& Sauermann, H. (2010). A taste for science? PhD scientists's academic orientation and self-selection into research careers in industry. Research Policy, 39(3), 422-434. doi:10.1016/j. respol.2010.01.004

Robinson, G., \& Dechant, K. (1997). Building a business case for diversity. The Academy of Management Executive, 11(3), 21-31.

Sarooghi, H., Libaers, D., \& Burkemper, A. (2015). Examining the relationship between creativity and innovation: A meta-analysis of organizational, cultural, and environmental factors. Journal of Business Venturing, 30(5), 714-731. doi:10.1016/j. jbusvent.2014.12.003

Sauermann, H., \& Cohen, W. (2008). What makes them tick? Employee motives and firm innovation. National Bureau of Economic Research, Working Paper No. 14443.

Shane, S., \& Venkataraman, S. (2000). The promise of entrepreneurship as a field of research. Academy of Management Review, 25(1), 217226.

Simonen, J., \& McCann, P. (2008). Innovation, R\&D cooperation and labor recruitment: Evidence from Finland. Small Business Economics, 31(2),181-194. doi:10.1007/s11187-007-9089-3

Simons, T., Pelled, L. H., \& Smith, K. A. (1999). Making use of difference: Diversity, debate, and decision comprehensiveness in top management teams. Academy of Management Journal, 42(6), 662673.

Slater, S. F., Mohr, J. J., \& Sengupta, S. (2014). Radical product innovation capability: Literature review, synthesis, and illustrative research propositions. Journal of Product Innovation Management, 31(3), 552-566. doi:10.1111/jpim.12113 
Stephan, P. E. (2006). Wrapping it up in a person: The mobility patterns of new PhDs. Innovation Policy and the Economy, 7, 71-98.

Torchia, M., Calabrò, A., \& Huse, M. (2011). Women directors on corporate boards: From tokenism to critical mass. Journal of Business Ethics, 102(2), 299-317. doi:10.1007/s10551-011-0815-Z

Unger, J. M., Rauch, A., Frese, M., \& Rosenbusch, N. (2011). Human capital and entrepreneurialsuccess entrepreneurial success: A metaanalytical review. Journal of Business Venturing, 26(3), 341-358. doi:10.1016/j.jbusvent.2009.09.004

Vegt, G. Van der, \& Janssen, O. (2003). Joint impact of interdependence and group diversity on innovation. Journal of Management, 29(5), 729-751. doi:10.1016/S0149-2063_03_00033-3

Velu, C. (2015). Business model innovation and third-party alliance on the survival of new firms. Technovation, 35, 1-11. doi:10.1016/j. technovation.2014.09.007

Wan, D., Ong, C., \& Lee, F. (2005). Determinants of firm innovation in Singapore. Technovation, 25(3), 261-268. doi:10.1016/S01664972(03)00096-8
Wiersema, M., \& Bantel, K. (1992). Top management team demography and corporate strategic change. Academy of Management Journal, 35(1), 91-121.

Williams, K., \& O’Reilly, C. (1998). Demograhpy Demography and diversity in organizations: A review of 40 years of research. Research in Organizational Behavior, 20, 77-140.

Wu, S. H., Lin, L. Y., \& Hsu, M. Y. (2007). Intellectual capital, dynamic capabilities and innovative performance of organisations. International Journal of Technology Management, 39(3/4), 279-296. doi:10.1504/IJTM.2007.013496

Zahra, S., Neubaum, D., \& Huse, M. (2000). Entrepreneurship and medium-size companies: Exploring the effects of ownership and governance systems. Journal of Management, 26(5), 947-976. doi:10.1016/S0149-2063(00)00064-7

Zhang, Y., \& Begley, T. (2011). Perceived organisational climate, knowledge transfer and innovation in China-based research and development companies. Internatioinal International Journal of Human Resource Management, 22(1), 34-56. doi:10.1080/0958519 2.2011 .538967 